\title{
Gys1 antisense therapy rescues neuropathological bases of murine Lafora disease
}

\section{Running head:}

Gys1 ASO therapy for Lafora disease

\section{Saija Ahonen ${ }^{1 \dagger}$, Silvia Nitschke ${ }^{1,2, \dagger}$, Tamar R. Grossman ${ }^{3}$, Holly Kordasiewicz ${ }^{3}$, Peixiang Wang ${ }^{1}$, Xiaochu Zhao $^{1}$, Dikran R. Guisso ${ }^{2}$, Sahba Kasiri ${ }^{2}$, Felix Nitschke ${ }^{2,4}$, Berge A. Minassian ${ }^{1,2 *}$}

${ }^{1}$ Program in Genetics and Genome Biology, The Hospital for Sick Children Research Institute, Toronto, ON M5G 0A4, Canada

${ }^{2}$ Division of Neurology, Department of Pediatrics, University of Texas Southwestern Medical Center, Dallas, TX 75390, USA

${ }^{3}$ Department of Antisense Drug Discovery, Ionis Pharmaceuticals, Carlsbad, California, USA.

${ }^{4}$ Department of Biochemistry, University of Texas Southwestern Medical Center, Dallas, TX 75390, USA

†These authors contributed equally

\section{Corresponding author:}

Dr. Berge Minassian

Division of Neurology, Department of Pediatrics

UT Southwestern Medical Center

5323 Harry Hines Boulevard

Dallas, TX 75390

Phone: 214-456-9831

Email: berge.minassian@utsouthwestern.edu 


\section{Abstract}

Lafora disease is a fatal progressive myoclonus epilepsy. At root, it is due to constant acquisition of branches that are too long in a subgroup of glycogen molecules, leading them to precipitate and accumulate into Lafora bodies, which drive a neuroinflammatory response and neurodegeneration. As a potential therapy, we aimed to downregulate glycogen synthase, the enzyme responsible for glycogen branch elongation, in the disease’s mouse models. We synthesized an antisense oligonucleotide (Gys1-ASO) that targets the mRNA of the brainexpressed glycogen synthase 1 gene (Gys1). We administered Gys1-ASO by intracerebroventricular injection and analyzed the pathological hallmarks of Lafora disease, namely glycogen accumulation, Lafora body formation, and neuroinflammation. Gys1-ASO prevented Lafora body formation in young mice that had not yet formed them. In older mice that already exhibited Lafora bodies, Gys1-ASO inhibited further accumulation, markedly preventing large Lafora bodies characteristic of advanced disease. Inhibition of Lafora body formation was associated with prevention of astrogliosis and strong trends towards correction of dysregulated expression of disease immune and neuroinflammatory markers. Lafora disease manifests gradually in previously healthy teenagers. Our work provides proof of principle that an antisense oligonucleotide targeting the GYS1 mRNA could prevent, and halt progression of, this catastrophic epilepsy.

Keywords: Lafora disease, antisense oligonucleotides, neuroinflammation, glycogen synthase, therapy

Abbreviations: GYS1 (glycogen synthase 1), ASO (antisense oligonucleotide), Ctrl-ASO (control ASO), ICV (intracerebroventricular), LB (Lafora body), PBS (phosphate-buffered saline), PASD (periodic acidSchiff diastase), SEM (standard error of mean), IC50 (half maximal inhibitory concentration), mo (month) 


\section{Introduction}

Lafora disease is a teenage-onset progressive myoclonus epilepsy that results from a disturbance of glycogen metabolism. Normally, glucose chains longer than 12 units start precipitating, yet glycogen with 55,000 glucose units is soluble. The latter is achieved through actions of two enzymes, the chain-elongating glycogen synthase and glycogen branching enzyme. These enzymes act in concert to generate molecules that are highly and symmetrically branched, with branches that are short and pointing away from each other, allowing the molecules to be permeated with water and soluble. ${ }^{1,2}$ In recent years two additional enzymes have been shown necessary for proper glycogen structure, namely the glycogen phosphatase laforin (EPM2A) and its interacting ubiquitin E3 ligase malin (EPM2B/NHLRC1). ${ }^{2}$ The mechanisms by which laforin and malin regulate glycogen structure remain unclear. What is clear is that loss of either's function results in slow but continuous generation of a portion of glycogen molecules with overlong branches, which, perhaps by winding around each other and extruding water, cause affected molecules to precipitate and over time accumulate into Lafora bodies (LBs). In the brain, by teenage years the accumulating LBs drive a progressive neuroinflammatory process, which at least in part underlies the disease's intractable epilepsy, neurodegeneration and dementia. ${ }^{2-4}$

The Epm $2 a^{-/}$and $E p m 2 b^{-/-}$Lafora disease mouse models recapitulate the primary pathologies of the disease, namely the glycogen branch over-lengthiness, accumulation of LBs, and neuroinflammation. Downregulating glycogen synthase by breeding Lafora disease mice with mice deficient of genes for the brainexpressed glycogen synthase isoform (Gys1) or of its activator proteins prevents these abnormalities. This effect was achieved both by complete or partial (30-50\%) reduction of brain GYS1 activity. ${ }^{5-11}$ Additionally, conditional knockdown of Gys1 after disease onset, i.e. after LBs have already appeared, halts further LB formation and attenuates neuroinflammation. ${ }^{12,13}$

In the present work we designed an antisense oligonucleotide (ASO) that targets Gys1 mRNA. Delivered into the CSF of Lafora disease mouse models, the ASO halted LB formation and reduced neuroinflammation. The results open a path to an ASO-based therapy for Lafora disease. 


\section{Materials and methods}

Mice and delivery of ASOs

$E p m 2 a^{-/-}$and $E p m 2 b^{-/-}$mouse models were described previously. ${ }^{5,6}$ Animal procedures were approved by the Toronto Centre for Phenogenomics or Ionis Pharmaceuticals Institutional Animal Care and Use Committees. Male and female mice were anesthetized with isofluorane, and, except where indicated otherwise, $300 \mu \mathrm{g}$ ASO in $10 \mu \mathrm{l}$ PBS were injected intracerebroventrically (ICV) per indicated schedule, alternating ventricles at successive time points. Stereotactic injection coordinates were $0.3 \mathrm{~mm}$ anterior/posterior (anterior to bregma), $1.0 \mathrm{~mm}$ to right or left medial/lateral and $-3.0 \mathrm{~mm}$ dorsal/ventral. Analgesic (Metacam $2 \mathrm{mg} / \mathrm{kg}$ ) was administered before and for two days post injections. Gys1-ASO sequence is 5'CATGCTTCATTTCTTTATTG-3'. Littermate controls were a no-target ASO, 5'CCTATAGGACTATCCAGGAA-3' (Ctrl-ASO), or PBS. Mice were sacrificed by cervical dislocation. One hemisphere was snap-frozen in liquid nitrogen for qRT-PCR and biochemical analyses, the other immersed in $10 \%$ neutral buffered formalin for histo- and immunohisto-pathology.

Gys1 expression analysis in mouse brain samples

For most trials, RNA was extracted with the Qiagen RNeasy Lipid Tissue Mini Kit (Qiagen, \#74804) and using a 1 cc syringe and $21 \mathrm{~g}$ needle for tissue homogenization. DNA was digested with DNase I (Thermo Scientific, \#EN0521). cDNA was synthesized from $1 \mu \mathrm{g}$ RNA using the iScript Advanced cDNA Synthesis Kit (Bio-Rad, \#1725037). For the 3-12 mo trial, tissue was homogenized in Ambion TRIzol (Thermo Fisher Scientific, \#15596018). Homogenate was loaded onto Phase Lock Gels (VWR, \#10847-802). After centrifugation the aqueous phase was loaded onto RNA columns (PureLink ${ }^{\mathrm{TM}}$ RNA Mini Kit, Thermo Fisher Scientific, \#12183025). On-column DNaseI digestion was performed using the PureLink ${ }^{\mathrm{TM}}$ DNase Set

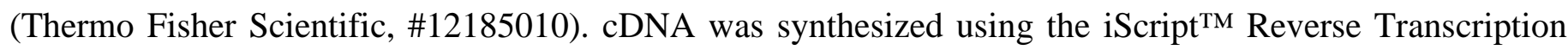
Supermix kit (Bio-Rad, \#1708841).

For qRT-PCR we used either the Stratagene Mx3005P (Agilent Technologies) or QuantStudio 7 Pro SmartStart (Applied Biosystems) real-time PCR system, and SYBR Green technology (PowerUp SYBR Green Master Mix [Applied Biosystems] or iTaq Universal SYBR Green Supermix [Bio-Rad]). Primer 
sequences were Gys1-F: 5'-CGCAAACAACTATGGGACAC-3', Gys1-R: 5'-

TCCTCCTTGTCCAGCATCTT-3'， Gapdh-F: 5'-AAGGGCTCATGACCACAGTC-3'， Gapdh-R: 5'-

GGATGCAGGGATGATGTTCT-3'， Hprt-F： 5'-TTGCTGACCTGCTGGATTAC-3'， Hprt-R: 5'-

ACTTTTATGTCCCCCGTTGA-3'， Rpl4-F: $\quad$ 5'-CCCTTACGCCAAGACTATGC-3'， Rpl4-R: $\quad$ 5'-

TGGAACAACCTTCTCGGATT-3'， Lcn2-F： 5'-GCCTCAAGGACGACAACATC-3'， Lcn2-R： 5'-

CACACTCACCACCCATTCAG-3'， Cxcl10-F： 5'-AAGTGCTGCCGTCATTTTCT-3'， Cxcl10-R： 5'AtAGGCTCGCAGGGATGATT-3'， Ccl5-F： 5'-TGCCAACCCAGAGAAGAAGT-3'， Ccl5-R： 5'AGCAAGCAATGACAGGGAAG-3'， C3-F： 5'-CTGTGTGGGTGGATGTGAAG-3'， and C3-R： 5'TCCTGAGTGTCGTTTGTTGC-3'. Gapdh served as reference, except for the 3-12 mo trial where Hprt and Rpl4 were used. $\Delta \mathrm{Ct}$ values were determined, calculating $\mathrm{Ct}_{\text {gene of interest }}-\mathrm{Ct}_{\text {reference gene }}$ (geometric mean was used in case of two reference genes), followed by transformation into $2^{-\Delta \mathrm{Ct}}$. Expression levels were further normalized to the PBS or Baseline group.

\section{Protein and glycogen analyses}

Frozen tissue was homogenized with buffer including Pierce protease and phosphatase inhibitors (Thermo Scientific) and $2 \mathrm{mM}$ DTT. Protein concentrations were determined using the DC Protein Assay (BioRad). Equal protein amounts $(30 \mu \mathrm{g})$ were heated in sample buffer $\left(70^{\circ} \mathrm{C}, 10 \mathrm{~min}\right)$ and loaded to $10 \% \mathrm{SDS}$-PAGE for Western blotting. GYS1 and GAPDH antibodies were from Cell Signaling (\#3886) and Santa Cruz (sc365062), respectively. Immunoblots were detected with HRP-conjugated secondary antibodies and Clarity Western ECL-substrate (BioRad) and analyzed with the ChemiDoc Imaging system (BioRad). Glycogen extraction, amyloglucosidase digestion and glucose determination were as previously described. ${ }^{14}$

\section{Histological analyses}

Formalin-fixed paraffin-embedded brain tissues were sectioned and stained using periodic acid-Schiff diastase (PASD) for $\mathrm{LBs}^{9}$ or immunohistochemistry against GFAP (mouse anti-GFAP, BioGenex, \#AM020-5M; dilution 1:250). Slides were scanned using Pannoramic (3DHistech) or NanoZoomer 2.0-HT (Hamamatsu 
Photonics) scanners. LB and GFAP signals were quantified in the hippocampus using HistoQuant (3DHistech) by defining LBs or GFAP signals based on pixel color. Values are expressed as \% area.

\section{Lafora body size distribution}

Per hippocampal section, HistoQuant analysis yielded in a list of individual LB sizes). LBs were assigned to 30 size bins with limits defined through a $3^{\text {rd }}$ order binomial function: lower limit $(n)=0.00222(n-1)^{3}+0.002$; upper limit $(\mathrm{n})=0.00222 \mathrm{n}^{3}+0.002 ; \mathrm{n}=$ bin number. The number of bodies per bin was divided by hippocampal area and expressed as area-normalized LB number $\left(\mathrm{n} / \mu \mathrm{m}^{2}\right)$. The average area-normalized body number per bin was calculated across all hippocampi of each group ( $\mathrm{n}>7$, biological replicates) and plotted against the bin center (average of lower and upper size bin limits). To calculate fold-change from baseline, area-normalized counts of each bin were divided by the average area-normalized count of the baseline group in the same bin (also performed with individual baseline values to obtain fold-changes to baseline average for each animal). Average fold-change and SEM were plotted against body size (bin center). Statistical analyses were performed comparing group average area-normalized body numbers and fold-changes for each bin.

\section{Statistical analyses}

Data are presented as mean \pm SEM. Statistics were performed with GraphPad Prism 8.4.3. If not stated otherwise, one-way ANOVA was performed, followed by post-hoc tests with Tukey multiple comparison correction. For Fig. 3A and B and Fig. 4E two-way ANOVA was performed, also followed by post-hoc tests with Tukey multiple comparison correction. For Fig. 3D and E one-way ANOVA was performed, followed by post-hoc tests with Holm-Bonferroni multiple comparison correction. For qRT-PCR data in Fig. 2 and Fig. 4 Kruskal-Wallis ANOVA was used, followed by two-stage linear step-up procedure of Benjamini, Krieger and Yekutieli. Asterisks denote statistical significance as follows: *, p <0.05; **, p $<0.01$; ***, $\mathrm{p}<0.001 ; * * * *, \mathrm{p}<0.0001$.

\section{Data availability}

The raw data that support the findings of this study are available from the corresponding authors, upon request. 


\section{Results}

\section{Identification of an active ASO}

We designed 300 ASOs targeting different regions of Gys1 pre-mRNA, which we screened for efficacy in mRNA downregulation. We selected Gys1-ASO for its potent (75\%), yet not complete, Gys1 mRNA downregulation (Supplementary Fig. 1A). The IC $_{50}$ of Gys1-ASO in cell-based experiments was $3.4 \mu \mathrm{M}$ (Supplementary Fig. 1A and B). We administered Gys1-ASO into ventricles of 1-month-old (mo) wild-type (WT) mice, sacrificed the mice 14 days later and measured Gys1 mRNA, which had diminished by greater than 50\% in the cortex, hippocampus and spinal cord (Supplementary Fig. 1C-E).

\section{Gys1-ASO prevents LB formation in young mice}

In the Lafora disease mouse models, LBs begin to form around 1 mo of age and become increasingly visible over the following two months, especially in the hippocampus. We delivered Gys1-ASO by ICV injection to

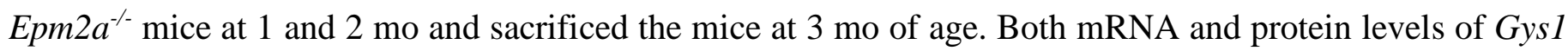
were reduced by greater than $80 \%$ in the Gys1-ASO-treated group (Fig. 1A-C). Brain glycogen levels are elevated in Lafora disease mice due to the accumulation of malstructured glycogen in the form of LBs. ${ }^{14}$ In Gys1-ASO-treated mice brain glycogen levels were more than $50 \%$ lower than in control mice (Fig. 1D) and comparable to levels normally seen in WT mice (approximately $2 \mu \mathrm{mol} / \mathrm{g}$ tissue fresh weight). ${ }^{14}$ Finally, PASD staining revealed that LBs were likewise and equivalently reduced (Fig. 1E and F). Results of these experiments in Epm2 $b^{-/}$mice were similar (Supplementary Fig. 2).

The above experiments showed that when administered at the point in time when LBs are starting to appear, Gys1-ASO inhibits LB accumulation. Results being similar using both mouse models, we limited subsequent experiments to the $E p m 2 a^{-/-}$genotype.

\section{Gys1-ASO prevents LB formation in older mice}

To determine whether the oligonucleotide is active in the context of pre-existing LB accumulations, we administered Gys1-ASO to older mice (3 mo and 8 mo old) with different specified dosing frequencies, doses and ages at sacrifice (Figs. 2 and 3A-C). These experiments were also designed in such a way as to inform us 
whether treatment with Gys1-ASO could reverse existing LB accumulations, i.e. not only prevent new accumulations, but lead to removal of existing ones. The results can be summarized as follows. Gys1-ASO does prevent LB accumulations beyond amounts present at time of treatment initiation. This effect is stronger when treatment starts earlier ( 3 mo versus $8 \mathrm{mo}$ ), is longer ( 3 mo to 12 mo versus 3 mo to 6 mo), or is dosed higher (500 $\mu$ g versus $300 \mu \mathrm{g}$ ) (Figs. 2 and 3A-C). LBs do not diminish below their existing levels.

\section{Gys1-ASO prevents a shift toward larger LBs}

LBs occur along a spectrum between two main morphologies, small and dust-like and large and spherical. The first are much smaller and much more numerous and are located in countless astrocytic processes. The second are mostly juxtanuclear in neuronal perikarya, occupying varying extents of neuronal cytoplasms. ${ }^{15,16}$ We studied the size distribution of hippocampal LBs in one of our experiments (3-12 mo trial) and show that across all sizes LB abundance increases with Lafora disease progression (PBS/Ctrl-ASO versus baseline), with larger LBs $\left(>1 \mu^{2}\right)$ contributing more to the overall increase of LBs (Fig. 3D and E). Gys1-ASO attenuated the growth of LBs of all sizes, in particular maintaining the abundance of larger bodies (1-60 $\left.\mu m^{2}\right)$ low compared to controls where large bodies markedly increased in number (Fig. 3E).

\section{Gys1-ASO prevents Lafora disease-related astrogliosis}

A remarkable $94 \%$ of genes upregulated in Lafora disease mouse model brain transcriptomes are ones involved in inflammatory and immune system pathways, strongly suggesting that immune disease at least in part underlies the disease. Of the hundreds of these genes a set of nine has been validated by qRT-PCR experiments, showing gradual increase in expression with Lafora disease progression. ${ }^{4}$ We measured expressions of four of these genes (Lcn2, Cxcl10, Ccl5, and C3) (as well as that of Gys1) in the 3-12 mo study and show that Gys1-ASO imparts a strong corrective tendency on the expression levels of all (Fig. 4A-E). We next studied whether microgliosis and astrogliosis, previously reported in murine Lafora disease, are improved. Aside from LBs, astrogliosis is the earliest and most constant neuropathological abnormality in LD mouse models. ${ }^{7-10,17}$ Microgliosis was not affected (Supplementary Fig. 3), but astrogliosis was corrected and near-eliminated (Fig. 4E-F). 


\section{Discussion}

Lafora disease afflicts previously healthy children with escalating and protracted devastation. A treatment is urgently needed. While the basic mechanisms of disease are not fully elucidated, the principal cog has recently become known, namely abnormally long branches, generated by glycogen synthase, leading to glycogen insolubility. ${ }^{3,18}$

ASOs have exceptional target specificities through Watson-Crick nucleotide sequence matching. Delivered to the CSF they distribute to practically all cells of all brain regions. They are stable and active for several months between doses. ${ }^{19}$ They can act through multiple mechanisms, e.g. activating a silent homologue (SMN2) of a mutated gene (SMN1) in spinal muscular atrophy, ${ }^{20,21}$ stabilizing and augmenting the activity of the healthy allele (SCN1A) in Dravet syndrome, ${ }^{22}$ and others. However, their original and simplest mechanism is downregulation of a target mRNA through RNaseH1, ${ }^{23}$ which is what is required in Lafora disease with GYS1 as target. We here show in the mouse models of Lafora disease that an ASO targeting Gys1 mRNA prevents the pathogenic LBs from forming or proliferating, and improves and corrects immunopathological features of the disease.

Mammals possess two glycogen synthase genes, one expressed exclusively in the liver (Gys2), the other (Gys1) in all the other organs including brain. ${ }^{24}$ Patients completely lacking GYS1 (glycogen storage disease type 0b) have no neurological disease but develop cardiac arrhythmias. Parents of these patients, with 50\% GYS1 activity, are completely healthy. ${ }^{25-27}$ A proposed therapy for Lafora disease with a GYS1 targeting ASO would be administered by lumbar puncture and thus not affect cardiac GYS1, as ASOs do not cross the blood-brain or arachnoid granulation barriers. ${ }^{28-30}$ In the brain, partial downregulation of GYS1 would be aimed for and sufficient. In previous studies based on genetic crosses of LD mice with mice deficient of GYS1 or proteins that activate GYS1, we and others showed that 30-50\% GYS1 downregulation suffices to prevent LB formation. ${ }^{9,10}$.

In the present work the ASO was effective at any disease stage, but more so in early disease and with sustained therapy. In the current era, LD patients can be diagnosed within weeks of symptoms. Myoclonic, visual or convulsive seizures lead to an EEG, which beyond epileptiform discharges shows background dysregulation and raises suspicion of a non-benign epilepsy. Since EPM2A and EPM2B are now widely 
included in epilepsy gene panels, diagnosis should then be swiftly made. In early stages of disease, LD patients are no different than other teenagers with new-onset epilepsy. As such, a therapy that halts the disease would be, in principle, tantamount to a cure.

Precisely how the laforin glycogen phosphatase and malin ubiquitin E3 ligase cooperate to fine-tune the lengths of glycogen branches to generate perfectly spherical and soluble macromolecules awaits to be uncovered. Meanwhile it is hoped that the present results will rapidly translate to a therapy for LD.

\section{Acknowledgments}

We would like to thank Jennifer P. Lee and Julia Gliwa for expert technical support.

\section{Funding}

This work was funded by the National Institutes of Health under award P01NS097197. S.A. was supported by the Sigrid Jusélius Foundation. B.A.M. holds the University of Texas Southwestern Jimmy Elizabeth Westcott Chair in Pediatric Neurology.

\section{Competing interests}

H.K. is a shareholder and employee at Ionis pharmaceuticals. T.R.G. has a patent (16/306831) pending. All other authors report no competing interests.

\section{Supplementary material}

Supplementary material is available at Brain online.

\section{References}

1. Roach PJ. Glycogen and its metabolism. Curr Mol Med. Mar 2002;2(2):101-20. doi:10.2174/1566524024605761

2. Nitschke F, Ahonen SJ, Nitschke S, Mitra S, Minassian BA. Lafora disease - from pathogenesis to treatment strategies. Nat Rev Neurol. Oct 2018;14(10):606-617. doi:10.1038/s41582-018-0057-010.1038/s41582-018-0057-0 [pii]

3. Sullivan MA, Nitschke S, Skwara EP, et al. Skeletal Muscle Glycogen Chain Length Correlates with Insolubility in Mouse Models of Polyglucosan-Associated Neurodegenerative Diseases. Cell Rep. Apr 30 2019;27(5):1334-1344 e6. doi:S22111247(19)30482-6 [pii]10.1016/j.celrep.2019.04.017

4. Lahuerta M, Gonzalez D, Aguado C, et al. Reactive Glia-Derived Neuroinflammation: a Novel Hallmark in Lafora Progressive Myoclonus Epilepsy That Progresses with Age. Mol Neurobiol. Mar 2020;57(3):1607-1621. doi:10.1007/s12035-019-01842-z10.1007/s12035-019-01842-z [pii] 
bioRxiv preprint doi: https://doi.org/10.1101/2021.02.11.430846; this version posted February 11, 2021. The copyright holder for this preprint (which was not certified by peer review) is the author/funder. All rights reserved. No reuse allowed without permission.

5. Ganesh S, Delgado-Escueta AV, Sakamoto T, et al. Targeted disruption of the Epm2a gene causes formation of Lafora inclusion bodies, neurodegeneration, ataxia, myoclonus epilepsy and impaired behavioral response in mice. Hum Mol Genet. May 16, 2002 2002;11(11):1251-1262. doi:10.1093/hmg/11.11.1251

6. Turnbull J, Wang P, Girard JM, et al. Glycogen hyperphosphorylation underlies Lafora body formation. Ann Neurol. Dec 2010;68(6):925-33. doi:10.1002/ana.22156

7. Pederson BA, Turnbull J, Epp JR, et al. Inhibiting glycogen synthesis prevents lafora disease in a mouse model. Annals of Neurology. 2013;74(2):297-300. doi:10.1002/ana.23899

8. Turnbull J, Epp JR, Goldsmith D, et al. PTG protein depletion rescues malin-deficient Lafora disease in mouse. Annals of Neurology. 2014;75(3):442-446. doi:10.1002/ana.24104

9. Turnbull J, DePaoli-Roach AA, Zhao X, et al. PTG depletion removes Lafora bodies and rescues the fatal epilepsy of Lafora disease. PLoS Genet. Apr 2011;7(4):e1002037. doi:10.1371/journal.pgen.1002037

10. Duran J, Gruart A, Garcia-Rocha M, Delgado-Garcia JM, Guinovart JJ. Glycogen accumulation underlies neurodegeneration and autophagy impairment in Lafora disease. Hum Mol Genet. Jun 15 2014;23(12):3147-56. doi:ddu024 [pii] 10.1093/hmg/ddu024

11. Israelian L, Nitschke S, Wang P, et al. Ppp1r3d deficiency preferentially inhibits neuronal and cardiac Lafora body formation in a mouse model of the fatal epilepsy Lafora disease. J Neurochem. Sep 6 2020;doi:10.1111/jnc.15176

12. Varea O, Duran J, Aguilera M, Prats N, Guinovart JJ. Suppression of glycogen synthesis as a treatment for Lafora disease: Establishing the window of opportunity. Neurobiol Dis. Nov 7 2020;147:105173. doi:S0969-9961(20)30448-4 [pii] 10.1016/j.nbd.2020.105173

13. Nitschke S, Chown EE, Zhao X, et al. An inducible glycogen synthase-1 knockout halts but does not reverse Lafora disease progression in mice. J Biol Chem. Dec 4 2020;doi:RA120.015773 [pii] 10.1074/jbc.RA120.015773 [doi]

14. Nitschke F, Sullivan MA, Wang P, et al. Abnormal glycogen chain length pattern, not hyperphosphorylation, is critical in Lafora disease. EMBO Mol Med. Jul 2017;9(7):906-917. doi:emmm.201707608 [pii] 10.15252/emmm.201707608

15. Augé E, Pelegrí C, Manich G, et al. Astrocytes and neurons produce distinct types of polyglucosan bodies in Lafora disease. Glia. Oct 2018;66(10):2094-2107. doi:10.1002/glia.23463

16. Rubio-Villena C, Viana R, Bonet J, et al. Astrocytes: new players in progressive myoclonus epilepsy of Lafora type. Hum Mol Genet. Apr 1 2018;27(7):1290-1300. doi:4835225 [pii] 10.1093/hmg/ddy044

17. López-González I, Viana R, Sanz P, Ferrer I. Inflammation in Lafora Disease: Evolution with Disease Progression in Laforin and Malin Knock-out Mouse Models. Mol Neurobiol. Jul 2017;54(5):3119-3130. doi:10.1007/s12035-016-9884-4 10.1007/s12035-016-9884-4 [pii]

18. Sullivan M, Nitschke S, Steup M, Minassian B, Nitschke F. Pathogenesis of Lafora Disease: Transition of Soluble Glycogen to Insoluble Polyglucosan. International Journal of Molecular Sciences. 2017;18(8):1743.

19. Geary RS, Norris D, Yu R, Bennett CF. Pharmacokinetics, biodistribution and cell uptake of antisense oligonucleotides. Adv Drug Deliv Rev. Jun 29 2015;87:46-51. doi:S0169-409X(15)00010-1 [pii] 10.1016/j.addr.2015.01.008 [doi]

20. Finkel RS, Mercuri E, Darras BT, et al. Nusinersen versus Sham Control in Infantile-Onset Spinal Muscular Atrophy. $N$ Engl J Med. Nov 2 2017;377(18):1723-1732. doi:10.1056/NEJMoa1702752

21. Mercuri E, Darras BT, Chiriboga CA, et al. Nusinersen versus Sham Control in Later-Onset Spinal Muscular Atrophy. $N$ Engl J Med. Feb 15 2018;378(7):625-635. doi:10.1056/NEJMoa1710504

22. Han Z, Chen C, Christiansen A, et al. Antisense oligonucleotides increase Scn1a expression and reduce seizures and SUDEP incidence in a mouse model of Dravet syndrome. Sci Transl Med. Aug 26 2020;12(558)doi:12/558/eaaz6100 [pii] 10.1126/scitranslmed.aaz6100

23. Crooke ST. Molecular Mechanisms of Antisense Oligonucleotides. Nucleic Acid Ther. Apr 2017;27(2):70-77. doi:10.1089/nat.2016.0656 [pii] 10.1089/nat.2016.0656 [doi]

24. Roach PJ, Depaoli-Roach AA, Hurley TD, Tagliabracci VS. Glycogen and its metabolism: some new developments and old themes. Biochem J. 2012;441:763-787. doi:10.1042/BJ20111416

25. Cameron JM, Levandovskiy V, MacKay N, et al. Identification of a novel mutation in GYS1 (muscle-specific glycogen synthase) resulting in sudden cardiac death, that is diagnosable from skin fibroblasts. Molecular Genetics and Metabolism. 2009;98(4):378-382. doi:http://dx.doi.org/10.1016/j.ymgme.2009.07.012

26. Kollberg G, Tulinius Mr, Gilljam T, et al. Cardiomyopathy and Exercise Intolerance in Muscle Glycogen Storage Disease 0. New England Journal of Medicine. 2007;357(15):1507-1514. doi:doi:10.1056/NEJMoa066691

27. Sukigara S, Liang WC, Komaki H, et al. Muscle glycogen storage disease 0 presenting recurrent syncope with weakness and myalgia. Neuromuscul Disord. Feb 2012;22(2):162-5. doi:S0960-8966(11)01336-8 [pii] 10.1016/j.nmd.2011.08.008

28. Geary RS, Yu RZ, Levin AA. Pharmacokinetics of phosphorothioate antisense oligodeoxynucleotides. Curr Opin Investig Drugs. Apr 2001;2(4):562-73.

29. Smith RA, Miller TM, Yamanaka K, et al. Antisense oligonucleotide therapy for neurodegenerative disease. J Clin Invest. Aug 2006;116(8):2290-6. doi:10.1172/JCI25424

30. Rinaldi C, Wood MJA. Antisense oligonucleotides: the next frontier for treatment of neurological disorders. Nat Rev Neurol. Jan 2017;14(1):9-21. doi:nrneurol.2017.148 [pii] 10.1038/nrneurol.2017.148 


\section{Figure legends}

Figure 1: Gys1-targeting ASO Gys1-ASO, administered at 1 and 2 months, leads to reduced Gys1 mRNA and GYS1 protein levels and attenuates glycogen and $\mathbf{L B}$ accumulation in $\boldsymbol{E p m}_{\mathbf{2}} \boldsymbol{a}^{-/-}$mice at $\mathbf{3}$ months. (A) Brain Gys1 mRNA relative expression levels in PBS-, Ctrl-ASO-, and Gys1-ASO-injected Epm2a-- mice. Ctrl-ASO, a no-target control ASO. (B) Brain GYS1 Western blots with GAPDH as loading control. (C) Quantification of GYS1 Western blots shown in B, normalized to GAPDH. (D) Brain total glycogen content. (E) Lafora body (LB) quantification in the hippocampus. (F) Representative images of PASD stained hippocampus. Scale bar, $50 \mu \mathrm{m}$. All data are presented as mean \pm SEM. Significance levels are indicated as *, p < 0.05; **, p < $0.01 ; * * *, \mathrm{p}<0.001 ; * * * *, \mathrm{p}<0.0001$.

Figure 2: Later ASO administration, after LD onset, effectively slows disease progression in Epm2a $^{-/-}$mice without signs of reversal. (A) Experimental design of three different trials. PBS, Ctrl-ASO (no-target control ASO), or Gys1-ASO were injected and mice sacrificed at indicated time points. Untreated mice, sacrificed at time of first injection, served as baseline control. (B to E) Results from 3-6 mo trial (trial design in A), showing Gys1 mRNA levels in Epm2a $a^{-/-}$(B), brain total glycogen content in Epm2a $a^{-/-}$ (C) and WT (D) and Lafora body (LB) quantification in the hippocampus of Epm2a $a^{-/-}$mice (E). (F to H) Results from 8-14 mo trial, using $300 \mu \mathrm{g}$ ASO for each injection (trial design in A), showing Gys1 mRNA levels (F), brain total glycogen content (G) and LB quantification in the hippocampus (H) of Epm2a $a^{-/-}$mice. (I and $\left.\mathbf{J}\right)$ Results from 8-14 mo trial, using a higher dose of $500 \mu \mathrm{gg}$ ASO for each injection (trial design in A), showing brain total glycogen content (I) and LB quantification in the hippocampus (J) of Epm2a $a^{-/-}$mice. (K) Representative images of PASD stained hippocampus of Epm $2 a^{-/-}$mice from the three different trials, explained in A. Scale bar, $50 \mu \mathrm{m}$. All data are presented as mean \pm SEM. Significance levels are indicated as *, $\mathrm{p}<0.05 ; * *, \mathrm{p}<0.01 ; * * *$, $\mathrm{p}$ $<0.001 ; * * * *, \mathrm{p}<0.0001$.

Figure 3: Long-term ASO treatment strongly prevents glycogen and LB accumulation in Epm2a ${ }^{-/}$mice. (A) Brain total glycogen content. (B) Lafora body (LB) quantification in the hippocampus. (C) Representative images of PASD stained hippocampus. Scale bar, $50 \mu \mathrm{m}$. PBS, Ctrl-ASO (no-target control ASO), or Gys1-ASO were injected at 3, 6, and 9 months and brain tissue analyzed at 12 months. Untreated mice, sacrificed and analyzed at 3 months, served as baseline control. Significance levels are indicated as *, p < 0.05; ***, $\mathrm{p}<0.001$; ****, $\mathrm{p}<0.0001$. Asterisks in pink indicate significance levels compared to the corresponding WT. (D) LB size distribution in Epm2a $a^{-/}$mice. (E) Differential LB size distribution (fold change compared to Baseline) in Epm2a $a^{-/}$mice. Top graph in D and E, p values comparing LB number between indicated experimental groups at different LB area bins. All data are presented as mean \pm SEM.

Figure 4: Long-term ASO treatment rescues astrogliosis in Epm2a ${ }^{-/-}$mice. (A to E), mRNA relative expression levels of Gys1 (A), and inflammatory and immune system response marker genes Lcn2 (B), Cxcl10 (C), Ccl5 (D), and C3 (E) analyzed by qRT- 
PCR. (F) GFAP signal quantification in the hippocampus. (G) Representative immunohistochemistry (IHC) images of anti-GFAP in the hippocampus. Scale bar, $50 \mu \mathrm{m}$. PBS, Ctrl-ASO (no-target control ASO), or Gys1-ASO were injected at 3, 6, and 9 months and brain tissue analyzed at 12 months. Untreated mice, sacrificed and analyzed at 3 months, served as baseline control. All data are presented as mean \pm SEM. Significance levels are indicated as *, p $<0.05 ; * *, p<0.01 ; * * *, p<0.001 ; * * * *, p<0.0001$. Asterisks in pink indicate significance levels compared to the corresponding WT. 
A

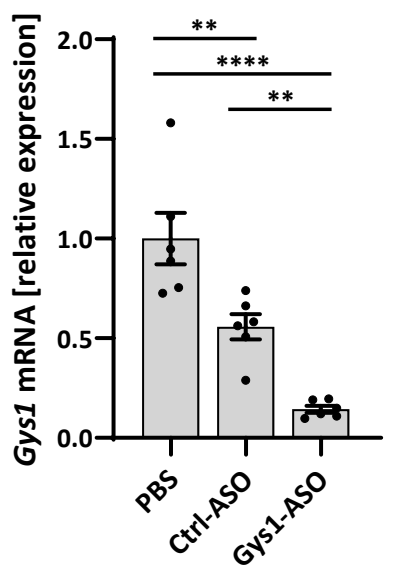

B

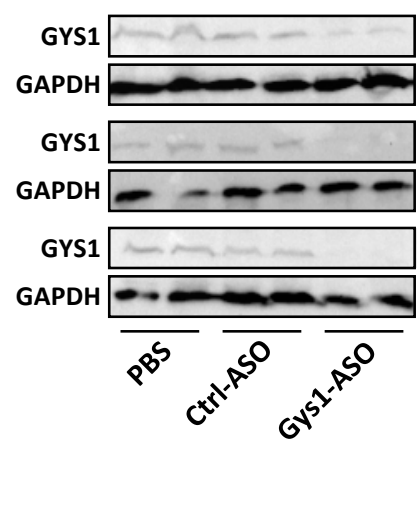

C

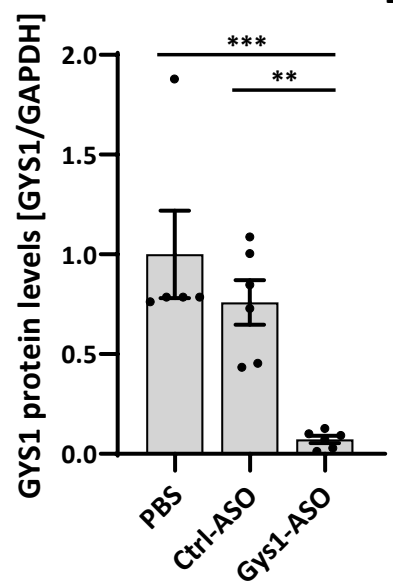

D

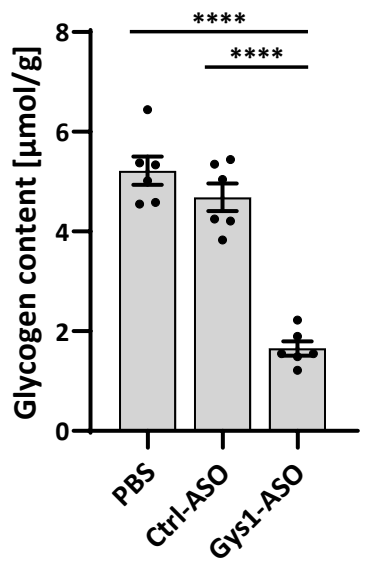

E

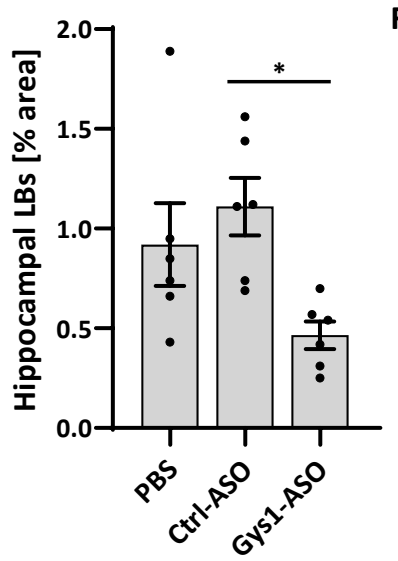

F

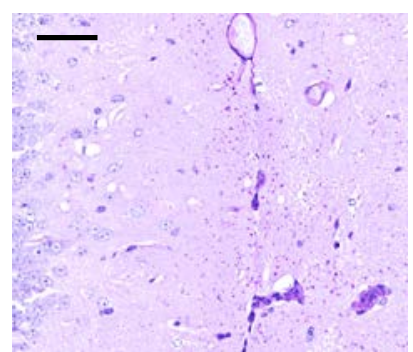

PBS

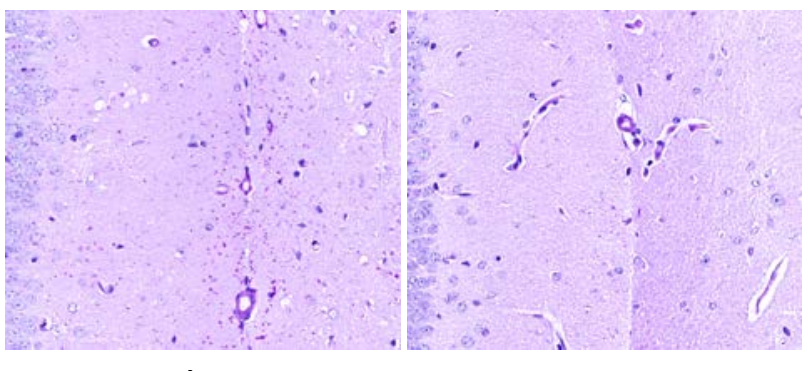

CtrI-ASO

Gys1-ASO

Figure 1: Gys1-targeting ASO Gys1-ASO, administered at 1 and 2 months, leads to reduced Gys1 mRNA and GYS1 protein levels and attenuates glycogen and LB accumulation in Epm2a $\%$ mice at 3 months. (A) Brain Gys1 mRNA relative expression levels in PBS-, Ctrl-ASO-, and Gys1-ASO-injected Epm $2 a^{-/}$mice. Ctrl-ASO, a no-target control ASO. (B) Brain GYS1 Western blots with GAPDH as loading control. (C) Quantification of GYS1 Western blots shown in B, normalized to GAPDH. (D) Brain total glycogen content. (E) Lafora body (LB) quantification in the hippocampus. (F) Representative images of PASD stained hippocampus. Scale bar, $50 \mu \mathrm{m}$. All data are presented as mean \pm SEM. Significance levels are indicated as $*, \mathrm{p}<0.05 ; * *, \mathrm{p}<0.01 ; * * *, \mathrm{p}<0.001 ; * * * *, \mathrm{p}<0.0001$. 
$\begin{array}{lll}\text { A } & 3-6 \text { mo trial B }\end{array}$

C

D

E

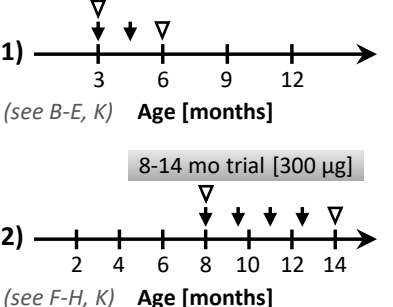

8-14 mo trial [500 $\mu \mathrm{g}]$
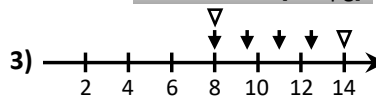
(see $I-K) \quad$ Age [months]

$\downarrow$ ICV injections $\nabla$ Harvest

$\mathbf{F}$

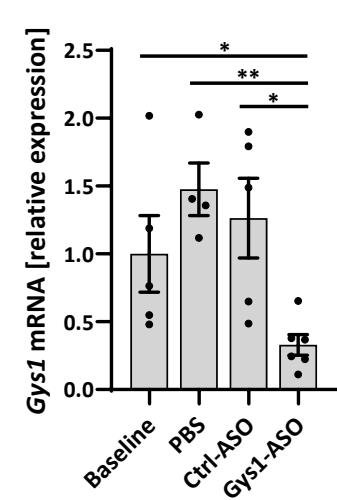

G
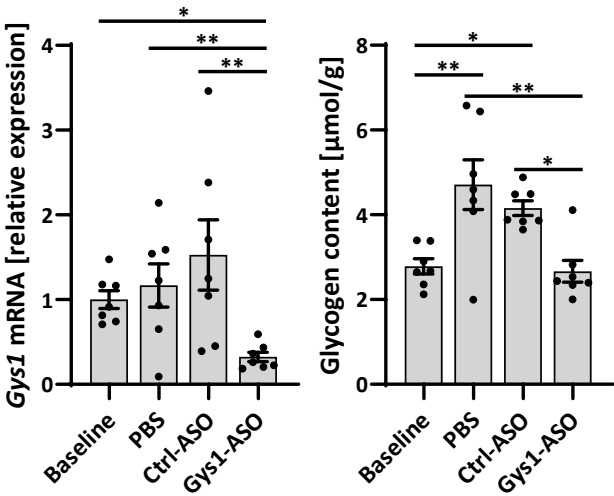

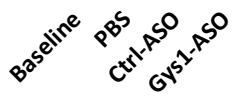

H
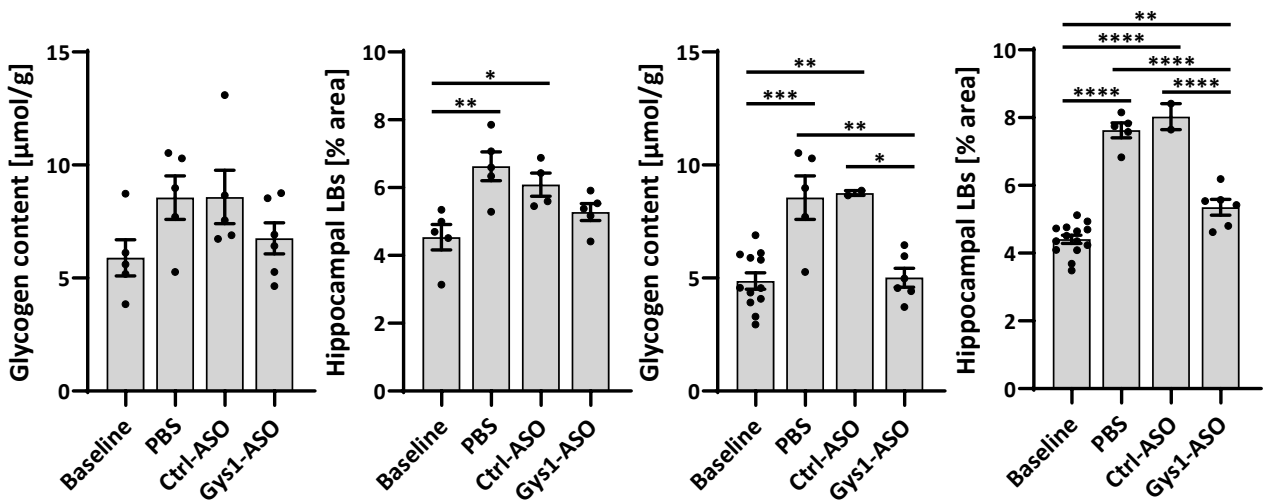

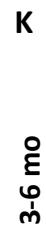

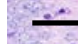

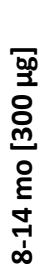

are:

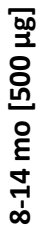
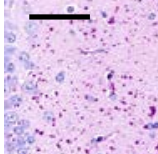

है.

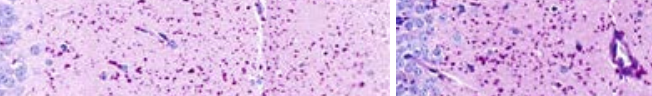


Figure 2: Later ASO administration, after LD onset, effectively slows disease progression in Epm2a/mice without signs of reversal. (A) Experimental design of three different trials. PBS, Ctrl-ASO (notarget control ASO), or Gys1-ASO were injected and mice sacrificed at indicated time points. Untreated mice, sacrificed at time of first injection, served as baseline control. (B to E) Results from 3-6 mo trial

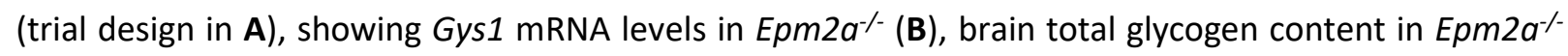
(C) and WT (D) and Lafora body (LB) quantification in the hippocampus of Epm2a $a^{-1}$ mice (E). (F to $\left.\mathbf{H}\right)$ Results from 8-14 mo trial, using $300 \mu \mathrm{g}$ ASO for each injection (trial design in A), showing Gys1 mRNA levels (F), brain total glycogen content $(\mathbf{G})$ and LB quantification in the hippocampus $(\mathbf{H})$ of Epm2a/mice. (I and J) Results from 8-14 mo trial, using a higher dose of $500 \mu \mathrm{g}$ ASO for each injection (trial design in A), showing brain total glycogen content (I) and LB quantification in the hippocampus (J) of Epm $2 a^{-/}$mice. (K) Representative images of PASD stained hippocampus of Epm $2 a^{-/}$mice from the three different trials, explained in A. Scale bar, $50 \mu \mathrm{m}$. All data are presented as mean \pm SEM. Significance levels are indicated as $*, p<0.05 ; * *, p<0.01 ; * * *, p<0.001 ; * * * *, p<0.0001$. 
A

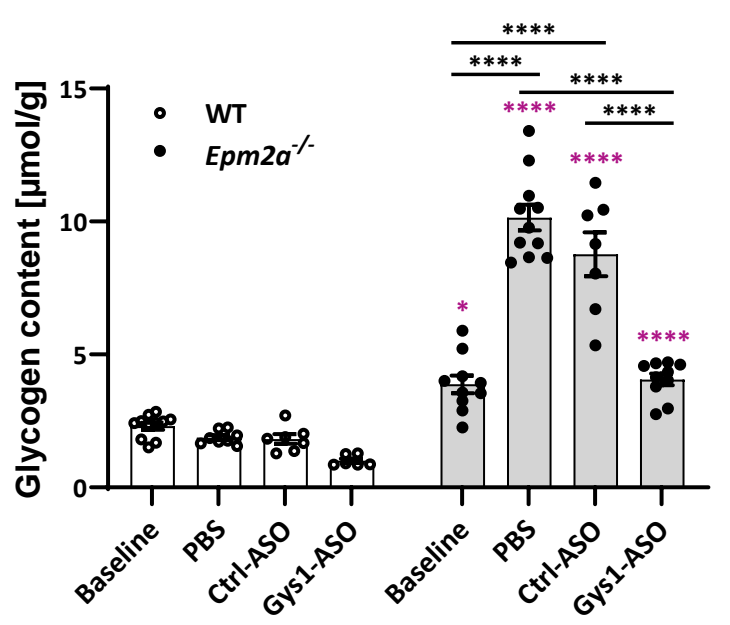

B

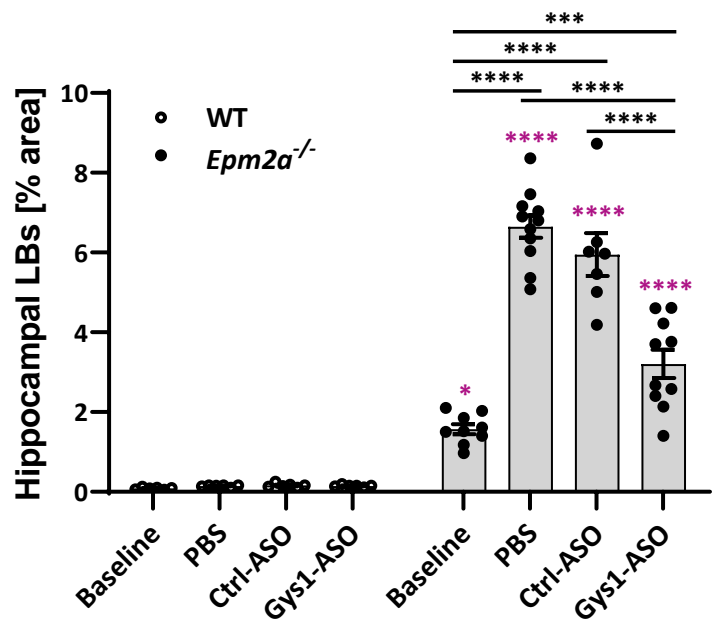

C
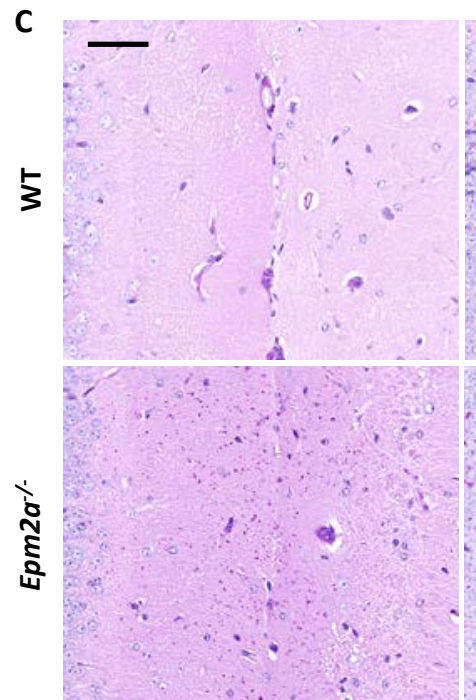

Baseline

PBS

Ctrl-ASO

Gys1-ASO

D
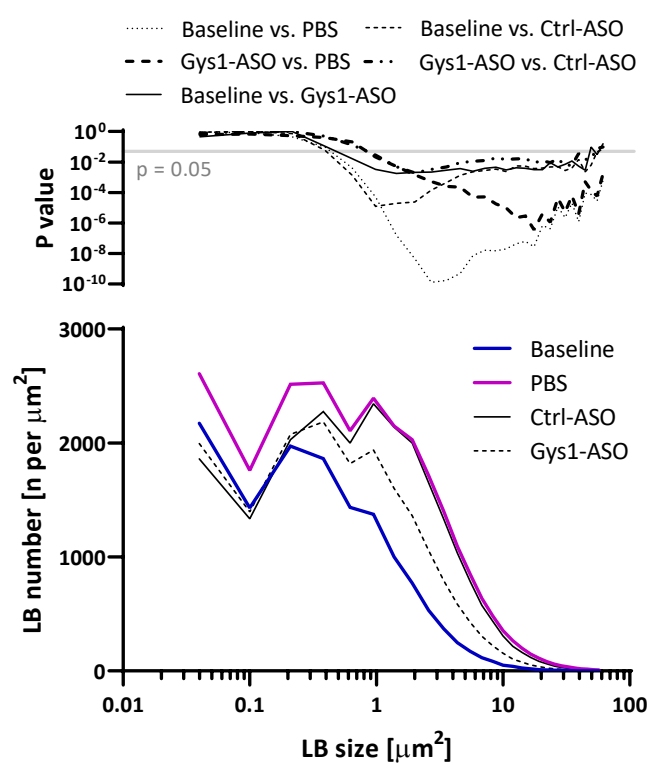

E
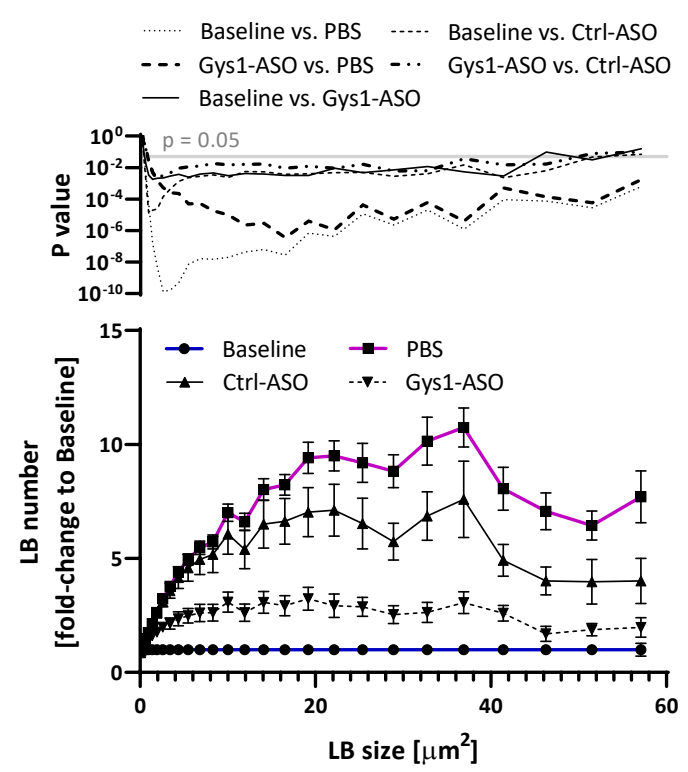

Figure 3 
Figure 3: Long-term ASO treatment strongly prevents glycogen and LB accumulation in Epm2a/mice. (A) Brain total glycogen content. (B) Lafora body (LB) quantification in the hippocampus. (C) Representative images of PASD stained hippocampus. Scale bar, $50 \mu \mathrm{m}$. PBS, Ctrl-ASO (no-target control ASO), or Gys1-ASO were injected at 3, 6, and 9 months and brain tissue analyzed at 12 months. Untreated mice, sacrificed and analyzed at 3 months, served as baseline control. Significance levels are indicated as $*, \mathrm{p}<0.05 ; * * *, \mathrm{p}<0.001 ; * * *, \mathrm{p}<0.0001$. Asterisks in pink indicate significance levels compared to the corresponding WT. (D) LB size distribution in Epm2a\%- mice. (E) Differential LB size distribution (fold change compared to Baseline) in Epm $2 a^{-/}$mice. Top graph in $D$ and $E, p$ values comparing LB number between indicated experimental groups at different LB area bins. All data are presented as mean \pm SEM. 
A

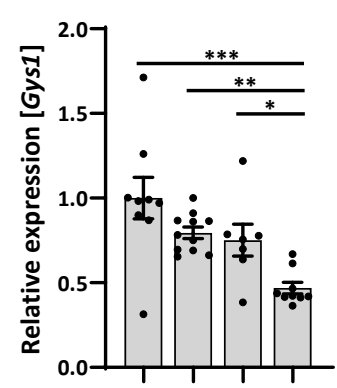

B

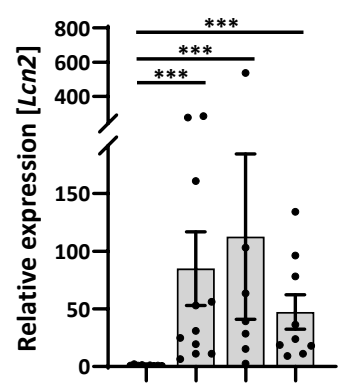

C

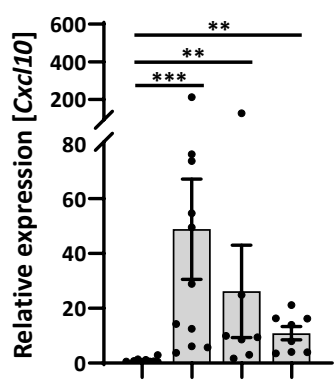

D

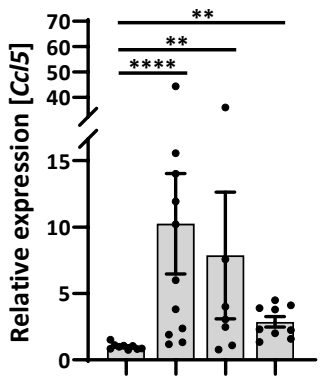

E

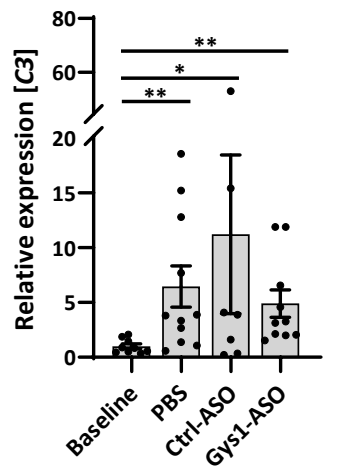

F

G

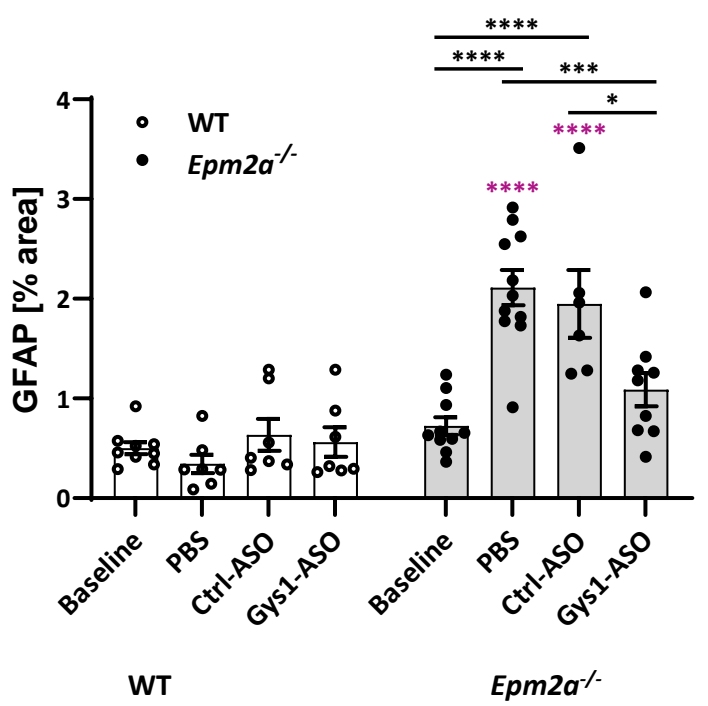

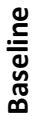

ֻั

Uั

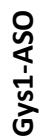


Figure 4: Long-term ASO treatment rescues astrogliosis. (A to E), mRNA relative expression levels of Gys1 (A), and inflammatory and immune system response marker genes Lcn2 (B), Cxcl10 (C), Ccl5 (D), and C3 (E) analyzed by qRT-PCR. (F) GFAP signal quantification in the hippocampus. (G) Representative immunohistochemistry (IHC) images of anti-GFAP in the hippocampus. Scale bar, $50 \mu \mathrm{m}$. PBS, Ctrl-ASO (no-target control ASO), or Gys1-ASO were injected at 3, 6, and 9 months and brain tissue analyzed at 12 months. Untreated mice, sacrificed and analyzed at 3 months, served as baseline control. All data are presented as mean \pm SEM. Significance levels are indicated as *, p < 0.05; **, p < 0.01; ***, p $<0.001$; $* * * *, p<0.0001$. Asterisks in pink indicate significance levels compared to the corresponding WT. 